\title{
Estado nutricional y aspectos alimentarios de mujeres indigenas del departamento de Presidente Hayes, Paraguay
}

\section{Nutritional state and food aspects of indigenous women from Presidente Hayes department, Paraguay}

\author{
Gloria Echagüe1, Valentina Díaz ${ }^{1}$, Laura Mendoza ${ }^{1}$, Pamela Mongelos ${ }^{1}$, Graciela Giménez ${ }^{1}$, Malvina Paez ${ }^{1}$, \\ Florentina Laspina ${ }^{1}$, Amalia Castro $^{1}$, María Isabel Rodríguez ${ }^{1}$, Patricia Araújo ${ }^{1}$, Wilberto Castro ${ }^{1}$, \\ Ramón Marecos², Santiago Evers², Gerardo Deluca³ ${ }^{3}$ Alejandra Picconi ${ }^{4}$.
}

Forma de citar: Echague G, Díaz V, Mendoza L, Mongelo P, Giménez G, Paez M, et al. Estado nutricional y aspectos alimentarios de mujeres indígenas del departamento de Presidente Hayes, paraguay. Rev Univ Ind Santander Salud. 2015; 47(3): 271-280. DOI: http://dx.doi.org/10.18273/revsal.v47n3-2015003 @c) (1) $\Theta$

\section{RESUMEN}

Introducción: Las comunidades indígenas presentan un mayor riesgo de inseguridad alimentaria y malnutrición, menor disponibilidad de recursos, y una creciente dependencia de alimentos más baratos aunque con un alto grado de procesamiento. Objetivo: Identificar el estado nutricional y aspectos alimentarios en mujeres indígenas de tres comunidades del Departamento de Presidente Hayes, Chaco Paraguayo. Metodología: Estudio observacional de diseño transversal con componente analítico, que incluyó a 81 mujeres de 15 a 44 años de edad, de las etnias Maká y Toba Qom. Previo consentimiento informado, se realizó la valoración nutricional y la entrevista para obtener los datos sociodemográficos y alimentarios. Resultados: Las mujeres indígenas presentaron una frecuencia de sobrepeso de 30,8\% y obesidad del $21,0 \%$, mientras que el $1,2 \%$ presentó desnutrición. No encontramos diferencia significativa entre las etnias, en relación al sobrepeso y obesidad, $(\mathrm{p}>0,05)$. Observamos que las indígenas tenían una alta ingesta de frutas, azúcares y mieles. El 87 y $88 \%$ de las mujeres indígenas de ambas etnias estudiadas refirieron no consumir lácteos y derivados y entre 65 y $69 \%$ refirieron no consumir verduras. Conclusiones: Observamos un elevado porcentaje de obesidad y sobrepeso, un bajo consumo de lácteos y verduras y un alto consumo de azúcares, relacionados posiblemente a cambios en los aspectos alimentarios y sus costumbres, influenciados por la cercanía a las zonas urbanas y el acceso a alimentos de menor precio y mayor contenido energético.

Palabras clave: estado nutricional, mujeres, alimentación.

1. Universidad Nacional de Asunción, Paraguay.

2. Hospital Regional de Villa Hayes - Ministerio de Salud Pública y Bienestar Social, Presidente Hayes, Paraguay.

3. Universidad Nacional del Nordeste. Corrientes, Argentina.

4. Instituto "Dr. Malbrán". Buenos Aires, Argentina.

Correspondencia: Gloria Echagüe. Dirección: Cecilio Báez, Campus Universitario, Universidad Nacional de Asunción. Email: gamechague@yahoo.com Teléfono: +59521920192 


\section{ABSTRACT}

Introduction: Indigenous communities present higher risk of food insecurity and malnutrition, lower availability of resources and growing dependence on cheaper food but with high degree of processing. Objective: To identify the nutritional state and food aspects in indigenous women from three communities of Presidente Hayes Department, Paraguayan Chaco. Methodology: Cross-sectional observational study with analytical component including 81 women who were 15 to 44 years old from the Maká and Toba Qom ethnic groups. After giving their informed consent, the nutritional assessment and the interview to collect socio-demographic and food data were carried out. Results: The indigenous women presented an overweight frequency of $30.8 \%$ and obesity of $21.0 \%$, while malnutrition was observed in $1.2 \%$. No significant differences were found between ethnic groups in relation to overweight and obesity ( $p>0.05$ ). High ingestion of fruits, sugar and honey was observed and $87 \%$ and $88 \%$ of the indigenous women from both ethnic groups referred that they did not consume dairy products and derivatives and $65 \%$ and $69 \%$ referred that they did not consume vegetables. Conclusions: High percentages of obesity and overweight were observed. Low consumption of dairy products, its byproducts and vegetables and a high consumption of sugar were observed, related probably to changes in food aspects and the habits of these groups, influenced by the proximity to urban areas and the access to low prices food with high energetic content.

Keywords: nutritional status, women, feeding.

\section{INTRODUCCIÓN}

Los pueblos indígenas de América Latina, tienden a ser pobres y a experimentar distintas áreas de vulnerabilidad superpuestas; no sólo carecen de lo indispensable, son frágiles en relación a salud, educación formal, igualdad de oportunidades, e influencia política ${ }^{1}$. De acuerdo a la Organización de las Naciones Unidas para la Agricultura y Alimentación (FAO), las comunidades indígenas presentan un mayor riesgo de inseguridad alimentaria y malnutrición que otros grupos, ya que padecen de niveles más elevados de pobreza, menor disponibilidad de recursos, y una creciente dependencia de alimentos más baratos aunque con un alto grado de procesamiento. La soberanía alimentaria de los pueblos indígenas se encuentra en riesgo por la erosión de los sistemas alimentarios tradicionales ${ }^{2}$. La malnutrición, o las condiciones físicas indeseables o de enfermedad que se relacionan con la nutrición, se pueden deber a comer muy poco, demasiado, o por una dieta desequilibrada que no contiene todos los nutrientes necesarios para un buen estado nutricional ${ }^{3}$. La distribución de las principales causas de mortalidad y morbilidad ha cambiado profundamente en los países desarrollados, y en muchos países en desarrollo se observa una tendencia similar. A nivel mundial ha aumentado rápidamente la carga de las enfermedades no transmisibles ${ }^{4}$. Un índice de masa corporal (IMC) de 25 era útil sólo para estimar el riesgo de muerte prematura, y esto se había convertido en el criterio para especificar su punto de corte; ahora sabemos que antes que el IMC aumente por encima de 25 , el riesgo de diabetes tipo 2 aumenta rápidamente entre un IMC de 20 y 25, así como el riesgo de presión arterial alta, de enfermedad isquémica del corazón y algunos tipos de cáncer relacionados con el incremento de peso ${ }^{5}$.

Las mujeres indígenas constituyen una población vulnerable; desde hace varios años y en especial desde la creación del Foro Permanente sobres Cuestiones Indígenas de la Organización de las Naciones Unidas (ONU), se lamenta la escasez de datos estadísticos sobre los pueblos indígenas, y los datos elaborados en los diferentes países confirman siempre la misma situación: los indígenas se encuentran en desventaja respecto a los no indígenas y las mujeres indígenas aún más que los hombres 6 .

En Paraguay, el estudio de Figueredo et al sobre la progresión de la obesidad en poblaciones indígenas, refiere que en las poblaciones nativas, la transculturación generada por las necesidades de subsistencia, las ha vuelto susceptibles a las afecciones metabólicas ${ }^{7}$. La pobreza en el país es 7,9\% veces mayor en los pueblos indígenas que en el resto de la población ${ }^{8}$. De acuerdo a los datos de la encuesta de hogares indígenas del 2008, las mujeres representan el 49,2 \% del total de la población indígena, el promedio del nivel educativo de la población, de 15 años o más, es de 3 años de estudio, y la tasa de analfabetismo en la población de 15 años o más, es de $38,9 \%{ }^{9}$. La literatura actual sobre mujeres indígenas es insuficiente. Las mujeres indígenas son más pobres, generalmente analfabetas, tienen mayor riesgo de morir a causa de enfermedades infecciosas y generalmente son víctimas de mortalidad perinatal ${ }^{8}$.

Las enfermedades no transmisibles, (ENT) enfermedad cardiovascular, cáncer, diabetes y enfermedad 
respiratoria crónica, son la principal causa de enfermedad y muerte prematura evitable en la Región de las Américas ${ }^{10}$. En Paraguay, ocupan las cuatro primeras causas generales de muerte desde hace varios años ${ }^{11}$. Según datos de la Primera Encuesta de Factores de Riesgo realizada en Paraguay en el 2011 en población indígena y no indígena, en mayores de 15 años, más de la mitad de la población adulta es obesa o presenta sobrepeso $^{12}$.

La Organización Panamericana de la Salud (OPS) y la Organización Mundial de la Salud (OMS) han presentado un plan de acción para la prevención y control de las ENT, donde la meta es la reducción del 25 \% como mínimo de la mortalidad prematura por ENT para el 2025, y una de las líneas estratégicas de acción es la vigilancia y estudios de investigación ${ }^{13}$.

Los indígenas del Paraguay están asentados predominantemente en las zonas rurales, (91,5\%); la mayor parte de la población indígena se encuentra en los departamentos de Presidente Hayes y Boquerón ${ }^{14,15}$. Cabe destacar que etnias, como los Maká y algunas comunidades de la etnia Toba Qom, tienen una presencia significativa en áreas urbanas o habitan próximos a los centros urbanos, como se observa en el departamento de Presidente Hayes. Esta distribución denotaría un desplazamiento hacía las ciudades, que podría intensificarse con los años. Las pirámides de la población indígena del país mantienen la forma característica de una estructura poblacional eminentemente joven, clara expresión de una fecundidad elevada, que luego se va reduciendo rápidamente en las edades adultas debido a las altas tasas de mortalidad a que están expuestas ${ }^{16,17}$, situación preocupante en especial entre las mujeres en edad reproductiva, más aún teniendo en cuenta que el departamento de Presidente Hayes se encuentra entre los cinco departamentos con las más altas tasas de mortalidad materna; el tercero con las tasas más altas de analfabetismo y el cuarto en base al índice de exclusión a las metas y objetivos del milenio, lo cual evidencia una mayor necesidad de asistencia, por sus mayores índices de exclusión en relación a las demás regiones ${ }^{18}$.

Los últimos estudios publicados en relación al estado nutricional de los indígenas del Chaco Paraguayo datan de los años 1998 al 2003, realizados en otras etnias indígenas del Chaco, en hombres y mujeres adultos de diferentes edades, asentadas en el departamento de Boquerón y zona norte y noroeste del departamento de Presidente Hayes, en comunidades alejadas de las ciudades departamentales ${ }^{19,20}$. Si bien se encuentra previsto el financiamiento en salud destinado a diferentes ciclos de vida de la población, entre ellas mujeres en edad fértil, por parte del Ministerio de Salud Pública y Bienestar Social ${ }^{21}$, el presupuesto asignado no alcanza a cubrir las necesidades, o no llega a las comunidades más vulnerables, exponiéndolas a una falta de asistencia sanitaria, actualmente no se cuenta con un programa activo y específico para mujeres en edad fertil ${ }^{22,23}$.

En algunas localidades donde la población indígena tiene una relación más estrecho con la sociedad envolvente, van apareciendo problemas de salud relacionados a los cambios en su estilo de vida y hábitos alimentarios, el problema de la tenencia de sus tierras, la migración a áreas urbanas, entre otros, constituyen nuevos desafíos para estas poblaciones, y hace necesario disponer de datos cuantificados, desagregados por comunidades sobre esta problemática. En el contexto de esta realidad, el objetivo de este estudio fue identificar el estado nutricional y aspectos alimentarios en mujeres indígenas en edad fértil que habitan próximas a las áreas urbanas del departamento de Presidente Hayes, debido al rol preponderante de las mismas en la reproducción y la lactancia, así mismo brindar datos actualizados que sirvan de base para tomar medidas estratégicas de control o prevención de la malnutrición en poblaciones vulnerables.

\section{METODOLOGÍA}

En el presente trabajo hemos estudiado a tres comunidades indígenas del departamento de Presidente Hayes, Chaco Paraguayo. La comunidad de Quemkuket, habitada por la etnia Maká, ubicada a 19 km de la capital departamental, la ciudad de Villa Hayes, y pertenece a la familia lingüística Mataco Mataguayo, no posee personería jurídica, se encuentran aún sin tierra propia (en trámite) ${ }^{24}$. La comunidad de Río Verde, habitada por la etnia Toba Qom, se encuentra a $17 \mathrm{~km}$ de la capital departamental, Villa Hayes, en el distrito de Benjamín Aceval, área rural, sin datos sobre la situación jurídica de sus tierras, y la comunidad Toba Qom, habitada por la etnia Toba Qom, se encuentra a $22 \mathrm{~km}$ de la capital departamental, Villa Hayes, en el distrito de Benjamín Aceval, área rural, posee personería jurídica y tierra propia; las indígenas de la etnia toba Qom pertenecen a la familia lingüística Guaicurú, Qom-Lik significa "Ser Humano", y "Toba" es llamado así por los guaraníes, por la costumbre de raparse la cabeza, lo que les dejaba a la vista una frente prominente ${ }^{25}$.

El diseño fue transversal con componente analítico, realizado en 81 mujeres indígenas de tres comunidades del Chaco Paraguayo, en el departamento de Presidente Hayes. 
De la comunidad de Quemkuket, participaron 38 mujeres de la etnia Maká, y de las comunidades de Río Verde y Toba Qom participaron 43 mujeres de la etnia Toba Qom, con un porcentaje de cobertura de la población total, en el periodo de estudio, del $51 \%$ $(\mathrm{n}=74)$ y $45 \%(\mathrm{n}=96)$ de las etnias Maká y Toba Qom respectivamente, la población no incluida se encontraba ausente en el momento del trabajo de campo.

El periodo de estudio fue entre el mes de octubre de 2010 y el mes de mayo de 2011. La población comprendió a todas las mujeres en edad reproductiva, de 15 a 44 años de edad ${ }^{26}$, que aceptaron participar del estudio, excluyendo a las mujeres embarazadas, mujeres lactantes o con tratamiento médico o quirúrgico durante el período de estudio. Para el reclutamiento se realizaron reuniones previas del equipo de trabajo acompañado por médicos y promotores de salud del Hospital Regional de Villa Hayes, el más cercano a éstas comunidades; inicialmente con el cacique y los líderes de la comunidad a fin de explicar el objetivo del trabajo; la segunda reunión, con las mujeres para informales el motivo del estudio y las condiciones requeridas para participar, y en la tercera visita a las comunidades, se procedió a la realización del trabajo de campo, en las mujeres que estaban de acuerdo en participar y reunían las condiciones para el estudio.

Las variables medidas fueron: edad, nivel de estudios, actividad a la que se dedica, ingreso mensual, fuente de abastecimiento de agua. La valoración del estado nutricional se realizó según el IMC, que considera el peso y la talla, utilizando instrumentos útiles para las mediciones, en uso en condiciones adversas de trabajo de campo, como son las comunidades indígenas ${ }^{27}$. Se registro el peso en kilogramos y se midió con una balanza mecánica marca Tokyo calibrada, de cero a 120 kilogramos, con precisión de $0,1 \mathrm{Kg}$, ajustada a cero antes de cada medición, la altura se midió con un tallímetro portátil fijado a la pared, tomada con la mujer descalza y en posición erguida, se registró la altura en metros. Los valores límites utilizados para el IMC fueron los recomendados por el Comité de Expertos de la Organización Mundial de la Salud (OMS/WHO) y la clasificación propuesta por el informe del comité de expertos WHO/FAO, Ginebra 2003 27,28:desnutrición severa IMC $<16 \mathrm{~kg} / \mathrm{m}^{2}$, desnutrición moderada IMC 16 a $16,9 \mathrm{~kg} / \mathrm{m}^{2}$, desnutrición leve IMC 17 a $18,4 \mathrm{~kg} / \mathrm{m}^{2}$, normopeso IMC 18,5 a $24,9 \mathrm{~kg} / \mathrm{m}^{2}$, sobrepeso grado I IMC 25 a $26,9 \mathrm{~kg} / \mathrm{m}^{2}$, sobrepeso grado II (preobesidad) IMC 27 a $29,9 \mathrm{~kg} / \mathrm{m}^{2}$, obesidad tipo I IMC 30 a $34,9 \mathrm{~kg} /$ $\mathrm{m}^{2}$, obesidad tipo II IMC 35 a $39,9 \mathrm{~kg} / \mathrm{m}^{2}$, obesidad tipo III (mórbida) IMC 40 a $49,9 \mathrm{~kg} / \mathrm{m}^{2}$, obesidad tipo IV (extrema) $\mathrm{IMC}>50 \mathrm{~kg} / \mathrm{m}^{2}$.
Para evaluar el aspecto alimentario de las participantes se utilizó el método cualitativo de la frecuencia de consumo de alimentos, realizado por personal capacitado por medio de entrevistas, para conocer la frecuencia semanal con que se consumen los diferentes grupos de alimentos, en base a las guías alimentarías del Paraguay ${ }^{29}$, y de acuerdo a la olla alimentaria, en la cual en el grupo 1 se encuentran: cereales, tubérculos y derivados, en el grupo 2: frutas, en el grupo 3: verduras, en el grupo 4: leche y derivados, en el grupo 5: carnes, legumbres y huevos, en el grupo 6: azúcares y mieles, en el grupo 7: aceites y grasas; así como indagar el número de comidas que realizan en el día y sus preferencias.

Análisis estadístico: Los datos fueron procesados usando estadística descriptiva y analítica por medio del programa Epiinfo versión 3.5.1 (CDC, Atlanta). Para evaluar las diferencias de proporciones entre los grupos se uso la prueba de chi-cuadrado. Los resultados se consideraron significativos a un intervalo de confianza del $95 \%(\mathrm{p}<0,05)$.

\section{RESULTADOS}

De las 81 mujeres indígenas estudiadas, 38 mujeres pertenecían a la comunidad de Qemkuket de la etnia Maká a (situada a $30 \mathrm{Km}$ de la capital, Asunción), 20 mujeres a la comunidad de Río Verde (situada a $52 \mathrm{Km}$ de la capital, Asunción) y 23 mujeres a la comunidad Toba-Qom (situada a $51 \mathrm{Km}$ de la capital, Asunción), ambas comunidades pertenecientes a la etnia TobaQom. La Figura 1 ilustra el mapa del Paraguay, en el cual se encuentra el Departamento de Presidente Hayes, incluyendo las comunidades estudiadas.

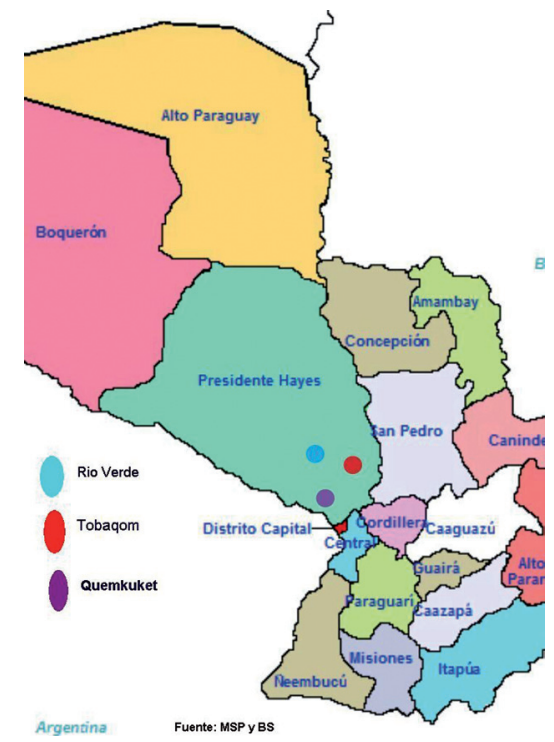

Figura 1. Mapa del Paraguay, Departamento de Presidente Hayes. 
Con respecto a las características de la población de mujeres estudiadas, la edad promedio fue de $30,6 \pm 11,7$ años, el promedio respecto al peso fue de $64,3 \pm 12,9$ $\mathrm{Kg}$, de la talla fue de 156,2 $\pm 0,061 \mathrm{~m}$ y del IMC fue de $26,4 \pm 5,1$. La tasa de analfabetismo fue de $29,6 \%$, la actividad a la que se dedicaban principalmente fue la artesanía. En relación al ingreso mensual la mayoría refirió no saber o no respondieron, seguido de las que refirieron no percibir ingreso y aquellas que percibían igual o menos de 500.000 guaraníes mensuales. La fuente de abastecimiento de agua fue mayoritariamente del tajamar. Las características sociodemográficas según etnia se observan en la Tabla 1 .

Tabla 1. Características sociodemográficas de las mujeres indígenas según etnia.

\begin{tabular}{|c|c|c|c|c|}
\hline \multirow[t]{2}{*}{$\begin{array}{c}\text { Variables } \\
\text { sociodemográficas }\end{array}$} & \multicolumn{2}{|c|}{ Etnia Maká } & \multicolumn{2}{|c|}{$\begin{array}{c}\text { Etnia Toba- } \\
\text { Qom }\end{array}$} \\
\hline & $\mathbf{n}$ & $\mathrm{IC}^{95 \%}$ & $\mathbf{n}$ & $\mathrm{IC}^{95 \%}$ \\
\hline \multicolumn{5}{|l|}{ Nivel de estudios (años) } \\
\hline 0 & 11 & $15,4-45,9$ & 13 & $17,2-46,1$ \\
\hline 1 a 6 & 24 & $46,0-78,2$ & 26 & $44,4-75,0$ \\
\hline 7 o más & 3 & $1,7-21,4$ & 4 & $2,6-22,1$ \\
\hline \multicolumn{5}{|l|}{ Ocupación } \\
\hline Estudiante & 2 & $0,6-17,7$ & 2 & $0,6-15,8$ \\
\hline Artesana & 27 & $54,1-84,6$ & 26 & $44,4-75,0$ \\
\hline Ama de Casa & 8 & $9,6-37,3$ & 8 & $8,4-33,4$ \\
\hline $\begin{array}{l}\text { Otros (agricultora, } \\
\text { servicios) }\end{array}$ & 1 & $0,1-13,8$ & 7 & $6,8-30,7$ \\
\hline \multicolumn{5}{|l|}{$\begin{array}{l}\text { Ingreso mensual } \\
\text { (en guaraníes) }\end{array}$} \\
\hline 0 a 500.000 & 19 & $33,4-66,6$ & 19 & $29,1-60,1$ \\
\hline Superior a 501.000 & 2 & $0,6-17,7$ & 1 & $0,1-12,3$ \\
\hline No sabe/No responde & 17 & $28,6-61,7$ & 23 & $37,7-68,8$ \\
\hline \multicolumn{5}{|c|}{ Abastecimiento de agua } \\
\hline Tajamar & 21 & $38,3-71,4$ & 20 & $31,2-62,3$ \\
\hline Pozo artesiano & 6 & $6,0-31,3$ & 10 & $11,8-38,6$ \\
\hline Red Pública & 11 & $15,4-45,9$ & 13 & $17,2-46,1$ \\
\hline
\end{tabular}

Los datos muestran que el problema nutricional más prevalente en las mujeres indígenas en edad fértil es el exceso de peso. El estado nutricional de la población de mujeres indígenas estudiadas presentó una frecuencia de sobrepeso de $30,8 \%$ y de obesidad del $21,0 \%$. El $1,2 \%$ de la población de mujeres indígenas presentó desnutrición. La mayor proporción de mujeres indígenas se encontraban en normopeso seguido por las de sobrepeso, como se indica en la Tabla 2.
Tabla 2. Estado nutricional de las mujeres indígenas estudiadas en el Departamento de Presidente Hayes, Paraguay.

\begin{tabular}{lccc}
\hline Estado nutricional & $\mathbf{n}$ & $\mathbf{\%}$ & IC $^{\mathbf{9 5} \%}$ \\
\hline Desnutrición Leve & 1 & 1,2 & $0,0-6,7$ \\
\hline Normopeso & 38 & 47,0 & $35,7-58,3$ \\
Sobrepeso & 25 & 30,8 & $21,1-42,1$ \\
\hline Obesidad & 17 & 21,0 & $12,7-31,5$ \\
Total & 81 & 100 & \\
\hline
\end{tabular}

Clasificación según WHO/FAO. Expert Consultation.World Health Organization, Geneva 2003.

La distribución del estado nutricional de las mujeres indígenas de acuerdo a la etnia a la que pertenecían se observa en la Tabla 3. Con respecto al sobrepeso observamos que las indígenas de la etnia Maká presentaron una frecuencia superior, de 31,6 \%, en relación a las mujeres indígenas de la etnia Toba-Qom; mientras que en relación a la obesidad, la etnia Toba Qom presentó una frecuencia superior de 27,9\%. Estas diferencias entre las etnias no fueron significativas, $(\mathrm{p}>0,05)$.

Tabla 3. Distribución del estado nutricional según etnia.

\begin{tabular}{|c|c|c|c|c|c|c|}
\hline \multirow{2}{*}{$\begin{array}{c}\text { Estado } \\
\text { Nutricional }\end{array}$} & \multicolumn{2}{|c|}{ Maká } & \multirow{2}{*}{$\mathrm{IC}^{95 \%} \%$} & \multicolumn{2}{|c|}{$\begin{array}{l}\text { Toba } \\
\text { Qom }\end{array}$} & \multirow{2}{*}{ IC ${ }^{95 \%}$} \\
\hline & $\mathbf{n}$ & $\%$ & & $\mathbf{n}$ & $\%$ & \\
\hline${ }^{\mathrm{a} D L}$ & 1 & 2,6 & $0,1-13,8$ & -- & -- & -- \\
\hline${ }^{\mathrm{b}} \mathrm{PN}$ & 20 & 52,6 & $35,8-69,0$ & 18 & 41,9 & $27,0-57,9$ \\
\hline${ }^{\mathrm{c}} \mathrm{SP}$ & 12 & 31,6 & $17,5-48,7$ & 13 & 30,2 & $17,2-46,1$ \\
\hline${ }^{\mathrm{d}} \mathrm{O}$ tipo & 5 & 13,2 & $4,4-28,1$ & 12 & 27,9 & $15,3-43,7$ \\
\hline
\end{tabular}

${ }^{\mathrm{a} D L}$ : desnutrición leve, ${ }^{\mathrm{b}} \mathrm{PN}$ : peso normal, ${ }^{\mathrm{c}} \mathrm{SP}$ : sobrepeso, ${ }^{\mathrm{d}} \mathrm{O}$ : obesidad

En relación al consumo de alimentos ingeridos, de acuerdo a la frecuencia de consumo en una semana, por grupo de alimentos, tanto las Maká como las Toba-Qom, se caracterizaron por presentar porcentajes similares de consumo de alimentos $(p>0,05)$. Las mujeres indígenas de las comunidades estudiadas tenían una baja ingesta de leche y derivados y una alta ingesta de frutas, azúcares y mieles, como se indica en la Figura 2.

El 65,8\% de las mujeres de la etnia Maká refirió realizar tres o más comidas al día (desayuno, almuerzo y cena), el 26,3\% dos comidas al día y sólo el 7,9 \% refirió realizar una comida en el día (desayuno o cena). El 60,5\% de las mujeres de la etnia Toba-Qom refirió realizar tres o más comidas al día (desayuno, almuerzo y cena), el 31,6\% dos comidas al día y sólo el 7,9\% refirió realizar una comida al día (desayuno o cena). 


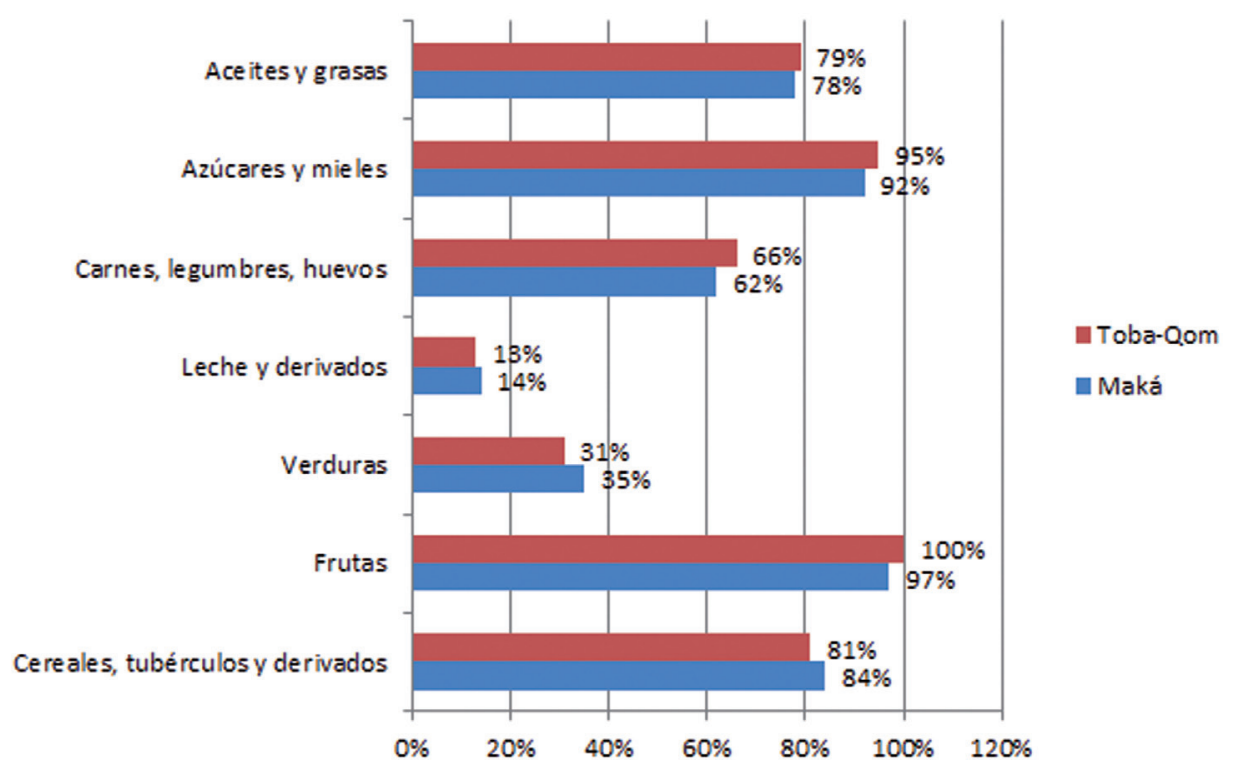

Figura 2. Porcentaje de mujeres indígenas de acuerdo a la ingesta por grupos de alimentos.

\section{DISCUSIÓN}

Con respecto al estado nutricional de las mujeres indígenas estudiadas cabe destacar el elevado porcentaje de obesidad y sobrepeso encontrados. En relación a estudios previos realizados en comunidades indígenas del país, relativo a la obesidad, observamos que en el trabajo publicado en el año 2000 por Vera Ruffinelli et $\mathrm{al}^{19}$ en una comunidad indígena de Sanapaná y Angaité, del Departamento de Presidente Hayes, Chaco Paraguayo, se encontró un 19,2\% de obesidad, con una significativa asociación al sexo femenino, en el año 2003 se publicó el trabajo realizado por Benítez et $\mathrm{al}^{20}$ en indígenas de la etnia Ayoreo de la colonia Carmelo Peralta del Departamento de Alto Paraguay, Chaco Paraguayo, en el cual se observó un 4 $\%$ de obesidad, de los cuales el 77,8 \% fueron del sexo femenino; en nuestro trabajo el porcentaje de obesidad encontrado en mujeres indígenas de las etnias Maká y Toba-Qom, fue superior a estos estudios previos (21 \%), y al reportado en la Encuesta Nacional de factores de riesgo en las mujeres indígenas en edades entre 15 y 74 años, de $17,3 \%{ }^{12}$; lo que podría sugerir un incremento de la obesidad en las etnias nativas, más aún teniendo en cuenta los resultados encontrados en los estudios previos mencionados.

Con relación al sobrepeso, el resultado observado en nuestro estudio en mujeres indígenas, de 30,8 \%, fue ligeramente superior al observado por Benítez et $\mathrm{al}^{20}$ en los Ayoreos, de $28,4 \%$, de los cuales el $62,5 \%$ correspondía al sexo femenino. Cabe destacar que el $51,8 \%$ de las mujeres indígenas estudiadas 276 en el presente trabajo presentaron sobrepeso $\mathrm{u}$ obesidad, frecuencia superior a la encontrada en la Primera Encuesta Nacional de Factores de Riesgo y Enfermedades no transmisibles en mujeres indígenas a nivel nacional de $42,7 \%$, mientras que más de la mitad de la población general de mujeres no indígenas presentaba un $60,7 \%$ de sobrepeso y obesidad ${ }^{12}$. Se podría sugerir que el incremento del sobrepeso y la obesidad encontrados en las comunidades indígenas estudiadas en el presente trabajo estén relacionadas con los cambios en los aspectos alimentarios de estas comunidades, así como de sus costumbres ancestrales, pudiendo deberse estos cambios en sus hábitos al contacto de las indígenas con las costumbres urbanas y el acceso a una alimentación de alto valor calórico y bajo precio, que encuentran en las ciudades cercanas, entre ellas la capital del país, donde van a vender sus artesanías.

Similares resultados a los encontrados en nuestras comunidades indígenas estudiadas fueron observadas por Ramos y et $\mathrm{al}^{30}$ en el estado de Oaxaca, México, en los indígenas Triquis, en la cual la proporción de sobrepeso y obesidad en mujeres fue del 50,8\%, y en su dieta tradicional se habían agregado alimentos densamente calóricos, como refrescos embotellados y harinas industrializadas.

En el estudio realizado por Rosique y et $\mathrm{al}^{31}$ en indígenas Embera de dos comunidades de Colombia, Embera oibida y Embera eyabida, en los adultos de ambos sexos, encontraron un mayor porcentaje de sobrepeso (45\%), con respecto a nuestros resultados 
y sólo un caso de obesidad, donde las mujeres se asociaron con mayor sobrepeso que los hombres; es posible que estas diferencias puedan deberse a los hábitos alimentarios de los Embera, los cuales aún se dedican a sus cultivos, a la caza y a la pesca. Cartes et $\mathrm{a}^{32}$ en el trabajo realizado en Chile en mujeres de la etnia Pehuenche en el 2011, observó niveles superiores para sobrepeso $(39,51 \%)$ y obesidad $(40,12 \%)$ que los obtenidos en nuestra población de mujeres indígenas Maká y Toba-Qom, posiblemente relacionados a la mayor accesibilidad de las indígenas pehuenche a los alimentos altamente calóricos y a las condiciones geográficas y medioambientales, sin embargo con respecto a la desnutrición encontramos datos similares en las indígenas pehuenche de $1,23 \%$.

Con respecto al aspecto alimentario, si bien las indígenas de las comunidades estudiadas continúan consumiendo frutas que se encuentran en sus aldeas, llama la atención el elevado consumo de cereales, tubérculos y derivados (maíz, mandioca, fideo, harina), de azúcares y mieles (caramelos, gaseosas, cocido, azúcar, miel de abeja y miel negra) y de aceites y grasas, de alto valor calórico. Por otro lado se observa un bajo consumo de verduras, lácteos y derivados; entre el 87 y el $88 \%$ de las mujeres indígenas de ambas etnias estudiadas refirieron no consumir lácteos y derivados y entre 69 y $65 \%$ refirieron no consumir verduras, debido posiblemente a que han ido dejado sus costumbres de cultivo y caza, y al costo para acceder a los productos lácteos; la mayoría se caracterizan por realizar por lo menos tres comidas diarias. Figueredo ${ }^{7}$ en el estudio sobre obesidad en poblaciones indígenas, menciona que el aumento de la obesidad viene de la mano con cambios ambientales, sedentarismo e incremento de la densidad calórica de los alimentos consumidos, determinando hábitos de consumo de tipo occidental.

En el estudio de los indígenas Embera ${ }^{31}$ se encontró baja ingesta de lácteos y verduras, similares a nuestros resultados, aunque un frecuente consumo de cereales y tubérculos, plátanos y productos cárnicos, mientras que nativos de nuestro estudio poseen una alta ingesta de aceites, grasas, y azúcares y mieles, relacionados posiblemente a los factores geográficos de los asentamientos de nuestros indígenas Maká y Toba-Qom, los cuales se encuentran más cercanos a las ciudades, lo que les permite mayor accesibilidad y contacto intercultural, mientras que los Embera se encuentran geográficamente más alejados, los indígenas Oibida de Atausí (indígenas de la selva) y los Eyabida de Nusidó (indígenas de las laderas).
Es importante considerar que el estado nutricional y los aspectos alimentarios de las comunidades indígenas estudiadas están relacionadas con las condiciones socioeconómicas de pobreza en las que viven, el nivel de educación, siendo el porcentaje de analfabetismo elevado (29,6\%), y los bajos ingresos que perciben en las actividades a las que se dedican, que en el caso de las mujeres de las etnias Maká y Toba-Qom del Departamento de Presidente Hayes, fue mayormente a la artesanía, seguido del cuidado de su hogar y en menor medida la prestación de servicios en estancias de la zona, a diferencia de los datos encontrados en el Departamento de Alto Paraguay y Boquerón en comunidades indígenas, en ambos sexos, en los cuales se observó que los indígenas se dedican principalmente a la agricultura, servicios y en tercer lugar a la $\operatorname{artesanía~}^{33}$, por lo que suponemos que podrían tener un mejor acceso a los alimentos de los cultivos de la zona, aunque no encontramos información al respecto.

Es de tener en cuenta que la tarea de obtener el agua recae principalmente en la mujer en las comunidades indígenas del Departamento de Presidente Hayes, y que la principal fuente de abastecimiento de agua fue el tajamar, tanto en la comunidad Maká como en las comunidades de la etnia Toba-Qom, en el cual no está garantizada la calidad del agua; siendo también ésta una diferencia con comunidades indígenas de los Departamentos de Alto Paraguay y Boquerón donde la fuente principal de agua es el pozo artesiano ${ }^{33}$.

En la actualidad hay que ampliar la mirada hacía las comunidades indígenas próximas a las áreas urbanas, cuya realidad es muy diferente al resto, donde se imponen acciones diferenciadas conforme a las características en las que habitan los indígenas, que afectan la Seguridad Alimentaria y Nutricional de sus comunidades, como el hábito de consumir comidas chatarras, alimentos que contienen conservantes, gaseosas o sintéticos ${ }^{16}$.

En el país no se han registrado mayores avances respecto del cumplimiento de las recomendaciones de la Asamblea General de las Naciones Unidas, Comité para la Eliminación de la Discriminación contra la Mujer, en donde, entre las observaciones finales al Paraguay se expresa, en referencia a la situación de las mujeres indígenas, una profunda preocupación sobre la alta vulnerabilidad en relación con el derecho a la alimentación adecuada y al agua segura, especialmente en la región del Chaco paraguayo ${ }^{34}$. 
El Sistema Nacional de Salud no cuenta con información desagregada que permita relevar evidencias de la situación de las comunidades indígenas, y existe una deficiencia del sistema de información en salud, que no contempla entre sus datos una diferenciación e identificación étnica de los casos registrados, a ello se suma la falta de aplicación de programas para mujeres en edad reproductiva no gestantes. Se encuentra entre los objetivos del sistema de salud, la promoción de la investigación y la creación de un sistema de información en salud indígena ${ }^{15}$.

De acuerdo al índice de exclusión basado en los objetivos y metas del milenio, desde el punto de vista de los sistemas de servicios de salud, el Departamento de Presidente Hayes se encuentra entre los que presentan los mayores déficits ${ }^{18}$. La carga de morbilidad causada por las enfermedades crónicas está aumentando rápidamente en Paraguay, y se estima que si no se toman medidas oportunas tendrá consecuencias sociales, económicas y sanitarias significativas ${ }^{12}$. A esto se suma el alto grado de pobreza y marginación de los indígenas, los cuales se desplazan hacia las zonas urbanas buscando oportunidades para vender sus artesanías o en muchos casos mendigan por las calles ${ }^{8,17}$.

No se han publicado estudios sobre el estado nutricional en mujeres en edad reproductiva de las etnias Maká y Toba-Qom asentados en el Chaco Paraguayo, los últimos estudios publicados realizados en otras etnias datan del año 2003; los hallazgos de este trabajo ponen de manifiesto el elevado porcentaje de sobrepeso y obesidad encontrados en las mujeres indígenas de las etnias estudiadas, aportando datos al sistema de salud en la identificación de los factores de riesgo, que permitan aplicar mecanismos de prevención o intervención en estas comunidades, y reducir los riesgos de morbimortalidad de las enfermedades no transmisibles, promoviendo la educación nutricional de acuerdo a su cultura y costumbres, más aún teniendo en cuenta la importancia del rol de la mujer en los hábitos alimentarios de su familia; a su futura función reproductiva potencial y las repercusiones de la mala nutrición de la mujer en su descendencia.

\section{CONCLUSIONES}

Más de la mitad de las mujeres indígenas en edad fértil se encontraban con sobrepeso u obesidad en el momento del estudio, presentando un marcado porcentaje para ambos estados nutricionales. La proporción de obesidad encontrada en las comunidades Maká y Toba Qom estudiadas fue superior al observado en estudios anteriores realizados en otras etnias localizadas en la zona central y norte del Chaco Paraguayo, lejos de los centros urbanos, lo que sugeriría que este problema de salud en las etnias nativas se va agudizando con el desplazamiento. Se consumían alimentos ricos en azúcares y se observó un bajo consumo de productos lácteos y verduras, relacionadas posiblemente a la disponibilidad de adquirir alimentos de menor precio y mayor contenido energético en las zonas urbanas, cerca de las cuales se asientan las comunidades estudiadas.

El trabajo realizado brinda datos al sistema de salud que permitirá a las autoridades sanitarias identificar la situación nutricional de las mujeres indígenas estudiadas y tomar medidas de prevención o intervención, con el fin de disminuir los riesgos de morbilidad y mortalidad, en especial por el importante rol de las mujeres en la reproducción, lactancia, el cuidado y la transferencia de hábitos y costumbres a su descendencia.

\section{AGRADECIMIENTOS}

Esta investigación fue posible gracias a la colaboración de la Técnica en Laboratorio Stella Mari Vázquez, a los docentes investigadores del Departamento de Análisis Clínicos del Instituto de Investigaciones en Ciencias de la Salud, a los profesionales de la XV Región Sanitaria del Departamento de Presidente Hayes y del Hospital Regional de Villa Hayes, a las mujeres participantes y a los líderes indígenas.

\section{CONSIDERACIONES ÉTICAS}

Se respetaron los tres principios éticos de autonomía, beneficio y justicia. Antes de realizar la evaluación antropométrica, y la entrevista para la obtención de los datos socio demográficos y alimentarios, las mujeres participantes firmaron un consentimiento informado aprobado por el Comité de Ética del Instituto de Investigaciones en Ciencias de la Salud de la Universidad Nacional de Asunción y los representantes indígenas acompañaron y fueron mediadores en todo el proceso del estudio. En el caso de las mujeres indígenas que hablaban dialectos, el consentimiento informado fue traducido a su idioma por miembros de su comunidad; y los padres o tutores de los menores de 18 años autorizaron la participación de los mismos. Todos los estudios realizados, en forma gratuita, fueron entregados al Hospital Regional de Villa Hayes, situado en el área de influencia de las localidades estudiadas, para el seguimiento y tratamiento de acuerdo al caso de las participantes del estudio. 
Estado nutricional y aspectos alimentarios de mujeres indígenas del departamento de Presidente Hayes, Paraguay

\section{DECLARACIÓN DE CONFLICTOS DE INTERESES}

Los autores declaran que no poseen ningún conflicto de intereses en relación al trabajo enviado para publicación. No existen compromisos ni obligaciones financieras con organismos estatales o privados de ningún tipo que puedan afectar el contenido, los resultados y las conclusiones de la presente publicación.

\section{REFERENCIAS}

1. Cimadamore AD, Eversole R, Mc Neish JA. Pobreza y pueblos indígenas: Una introducción a los enfoques multidisciplinarios. 1ed. Buenos Aires: CLACSO; 2006.

2. Organización de las Naciones Unidas para la Agricultura y Alimentación. Política de la FAO sobre pueblos indígenas y tribales. Roma: FAO; 2012.

3. Latham MC. Causas de la malnutrición: Nutrición internacional y alimentarios mundiales en perspectiva. Nutrición humana en el mundo en desarrollo. Roma: FAO; 2002.

4. Organización Mundial de la Salud. Estrategia mundial sobre régimen alimentario, actividad física y salud. Ginebra: OMS; 2004.

5. Philip J. All-of-government approach needed to tackle obesity. Bull World Health Organ. 2013; 91(8): 551-552.

6. Meentzen A. Estrategias de desarrollo culturalmente adecuadas para mujeres indígenas. Washington, D.C.: Banco Interamericano de Desarrollo; 2001.

7. Figueredo R, Vera Ruffinelli J, Benítez A, Bueno E. Progresión de la obesidad en poblaciones indígenas de Paraguay. Rev Esp Obes. 2007; 5(2): 91-97.

8. Pero Ferreira AM. Nota técnica de país sobres cuestiones de los pueblos indígenas: República del Paraguay. Asunción: Fondo Internacional de Desarrollo Agrícola; 2012.

9. Dirección General de Estadística, Encuestas y Censos (DGEEC). Paraguay principales resultados EHI/ 2008: Encuesta de hogares indígenas, características demográficas, sociales y económicas. Fernando de la Mora: DGEEC; 2008.

10. Organización Panamericana de la Salud, Organización Mundial de la Salud. Estrategia regional y plan de acción para un enfoque integrado sobre la prevención y el control de las enfermedades crónicas. Washington, DC: OPS; 2007.

11. Ministerio de Salud Pública y Bienestar Social, Organización Panamericana de la Salud, Organización Mundial de la Salud, Agencia Canadiense de Desarrollo Internacional. Indicadores básicos de salud. Asunción: MSP y BS; 2012.

12. Cañete F. Primera encuesta nacional de factores de riesgo y enfermedades no transmisibles: Paraguay 2011. Asunción: MSP y BS. Dirección Vigilancia de Enfermedades No Transmisibles; 2012.

13. Organización Panamericana de la Salud. Plan de acción para la prevención y control de las enfermedades no transmisibles en las Américas 2013-2019. Washington, DC: OPS; 2014.

14. Servin J, Bazán R, Royg P, Britez A, Otazú N. III Censo nacional de población y viviendas para pueblos indígenas: Pueblos indígenas en el Paraguay resultados preliminares 2012. Fernando de la Mora: DGEEC; 2013.

15. Ortega Rolón S, Cabral de Bejarano S, Medina L, Vera I, Espínola A, Peralta R, et al. Política nacional de salud indígena. Asunción: Ministerio de Salud Pública y Bienestar Social. Coordinadora Nacional de Pastoral Indígena. Fondo de las Naciones Unidas para la Infancia; 2008.

16. Ramos Rodas N. Informe nacional: Diagnóstico y propuestas para el desarrollo de una política pública de seguridad alimentaria y nutricional de los pueblos indígenas en Paraguay. Asunción: Ministerio de Agricultura y Ganadería. Instituto Paraguayo del Indígena, Organización de las Naciones Unidas para la Alimentación y la Agricultura; 2014.

17. Dirección General de Estadísticas, Encuestas y Censos (DGEEC). II Censo nacional indígena de población y viviendas 2002: Pueblos indígenas del Paraguay. Resultados Finales Fernando de la Mora: DGEEC; 2003.

18. Ojeda I, Vidal G, Cabral S, Godoy I, Gaete R. Focalización geográfica: Índice de exclusión basado en las metas del milenio: Metodología de selección. Asunción: Ministerio de Salud Pública y Bienestar Social. Dirección General de Planificación y Evaluación; 2004.

19. Vera de Ruffinelli J, Benítez A, Rojas de Arias A, Medina U, Almirón M, Russomando G, et al. Detección de diabetes tipo $2 \mathrm{y}$ otros factores de riesgo cardiovascular en una comunidad indígena de Sanapaná y Angaité del Chaco Paraguayo. Estudio preliminar. Rev ALAD. 2000; 8: 86-91.

20. Benítez A, Vera J, Arias A, Echagüe G, Moreno Azorero R. Obesidad, diabetes y otros factores de riesgo cardiovascular en los Ayoreo del Chaco Paraguayo. Rev ALAD. 2003; 11: 135-140.

21. Ministerio de Salud Pública y Bienestar Social, Dirección General de Planificación y Evaluación. Organización Panamericana de la Salud, Organización Mundial de la Salud. Financiamiento 
y gasto del Ministerio de Salud Pública y Bienestar Social por función de atención y ciclo de vida 2005/2006. Asunción: MSP y BS; 2007.

22. Ministerio de Salud Pública y Bienestar Social. Dirección General de Estadísticas Encuestas y Censos. Organización Panamericana de la Salud. Organización Mundial de la Salud. Exclusión social en salud. Asunción: MSP y BS; 2003.

23. Guillén MC. Sistemas de salud en Sudamérica: desafíos hacia la integralidad y equidad. Asunción: MSP y BS; 2011.

24. Dirección General de Encuestas, Estadística y Censo. Atlas de las comunidades indígenas en el Paraguay. Fernando de la Mora: DGEEC; 2004.

25. Terán B. La visión del Kiyok en el mundo Toba. Suplemento antropológico. 1984; 19(2): 169-208.

26. Organización Mundial de la Salud. Las mujeres y la salud: Los datos de hoy la agenda del mañana. Ginebra: OMS; 2009.

27. Organización Mundial de la Salud. El estado físico: uso e interpretación de la antropometría: Reporte de un Comité de Expertos de la OMS. Ginebra: OMS; 1995. Serie de Informes Técnicos 854.

28. World Health Organization. Diet nutrition and the prevention of chronic diseases. WHO/FAO: Expert Consultation. Ginebra: WHO; 2003. Technical Report Series 916.

29. Redondo Martinez JV, Pedotti Vázquez RE, Serafin P. Guías alimentarias para la población paraguaya. Asunción: Instituto Nacional de Alimentación y
Nutrición. Comité técnico nacional de las guías alimentarias del Paraguay; 2011.

30. Ramos Rodríguez RM, Sandoval Mendoza K. Estado nutricional en la marginación y la pobreza de adultos triquis del estado de Oaxaca, México. Rev Panam Salud Pública. 2007; 22(4): 260-267.

31. Rosique J, Restrepo MT, Manjarrés LM, Gálvez A, Santa J. Estado nutricional y hábitos alimentarios en indígenas Embera de Colombia. Rev Chil Nutr. 2010; 37(3): 270-280.

32. Cartes Velásquez $\mathrm{C}$, Navarrete Briones C. Caracterización antropométrica de población de Pehuenche adulta, consideraciones nutricionales Alto Biobio, Chile. Mem Inst Investig Cienc Salud. 2012; 8(2): 30-37.

33. Programa de las Naciones Unidas para el Desarrollo. Programa Conjunto Gobernabilidad en Agua y Saneamiento, Facultad de Ciencias Económicas, Universidad Nacional de Asunción. Análisis de la encuesta comunitaria indígena sobre agua potable y saneamiento 2009: Alto Paraguay - Boquerón. Asunción: PNUD; 2011.

34. Ramírez Ozorio EA. A pesar de un marco negativo propicio y específico el derecho a la alimentación de las personas indígenas continúa siendo vulnerado: El Derecho a la alimentación adecuada negado a los pueblos indígenas del Chaco. En: Gauto Bozzano E, coord. Derechos humanos en Paraguay 2013. 1 ed. Asunción: Coordinadora de Derechos Humanos del Paraguay; 2013. 